\title{
Predicting the potential distribution of the Asian citrus psyllid, Diaphorina citri (Kuwayama), in China using the MaxEnt model
}

\author{
Rulin Wang \\ Jinpeng Zhao ${ }^{2}$, Qing Li ${ }^{\text {Corresp. } 1}$ \\ Hua Yang ${ }^{\text {Equal first author, } 3}$, Wei Luo ${ }^{4}$, Mingtian Wang ${ }^{5,6}$, Xingli Lu $^{2}$, Tingting Huang ${ }^{1}$, \\ Sichuan Agricultural University, College of Agronomy, Chengdu, Sichuan, China \\ Sichuan Provincial Rural Economic Information Center, Chengdu, Sichuan, China \\ ${ }^{3}$ Sichuan Agricultural University, Key Laboratory of Ecological Forestry Engineering of Sichuan Province/College of Forestry, Chengdu, Sichuan, China \\ 4 Zigong Bureau of Meteorology, zigong, Sichuan, China \\ 5 Sichuan Meteorological Observatory, Chengdu, Sichuan, China \\ 6 Water-Saving Agriculture in Southern Hill Area Key Laboratory of Sichuan Province, Chengdu, Sichuan, China \\ Corresponding Authors: Rulin Wang, Qing Li \\ Email address: 398927566@qq.com, 10100@sicau.edu.cn
}

Background. Citrus huanglongbing (HLB) is a destructive disease of citrus and a major threat to the citrus industry around the world. This disease accounts for substantial economic losses in China every year. Diaphorina citri Kuwayama is one of the major vectors by which citrus HLB is spread under natural conditions in China. Research is needed to identify the geographic distribution of $D$. citri and its major areas of occurrence and to formulate measures for early warning, monitoring and control of this pest and citrus HLB.

Methods. In this study, the ecological niche modelling software MaxEnt (maximum entropy model) was combined with ArcGIS (a geographic information system) to predict the potential geographic distribution of $D$. citri in China. Key environmental factors and the appropriate ranges of their values were also investigated.

Results. Our results show that the training data provided a good forecast $\left(A \cup C_{\text {mean }}=0.988\right)$. The highly suitable areas for $D$. citri in China are mainly concentrated to the south of the Yangtze River, and the total area is $139.83 \times 10^{4} \mathrm{~km}^{2}$. The area of the moderately suitable areas is $27.71 \times 10^{4} \mathrm{~km}^{2}$, with a narrower distribution than that of the highly suitable area. The important environmental factors affecting the distribution of $D$. citri were Min Temperature of Coldest Month, Mean Temperature of Coldest Quarter, Precipitation of Wettest Quarter, Mean Temperature of Warmest Quarter, Precipitation of Warmest Quarter, Max Temperature of Warmest Month, and Temperature Seasonality. These results provide a valuable theoretical basis for risk assessments and control of $D$. citri.

Discussion. The predicted results showed that there were highly suitable areas for $D$. citri in Chongqing, Hubei, Anhui and Jiangsu. Therefore, the possibility exists for the further spread of $D$. citri in China in the future. Extreme temperature variables, especially the Min temperature of the coldest month, play an important role in the distribution of $D$. citri and are most closely related to the distribution of $D$. citri. 
5 Rulin Wang ${ }^{1,2}$, Hua Yang ${ }^{3}$, Wei Luo ${ }^{4}$, Mingtian Wang ${ }^{5,6}$, Xingli Lu ${ }^{1}$, Tingting Huang ${ }^{1}$, Jinpeng $6 \mathrm{Zhao}^{2}$, Qing $\mathrm{Li}^{1}$

$7{ }^{1}$ College of Agronomy, Sichuan Agricultural University, Chengdu, Sichuan, China

$8{ }^{2}$ Sichuan Provincial Rural Economic Information Center, Chengdu, Sichuan, China

$9{ }^{3}$ Key Laboratory of Ecological Forestry Engineering of Sichuan Province/College of Forestry, 10 Sichuan Agricultural University, Chengdu, Sichuan, China

4 Zigong Bureau of Meteorology, Zigong, Sichuan, China

${ }^{5}$ Sichuan Meteorological Observatory, Chengdu, Sichuan, China

${ }^{6}$ Water-Saving Agriculture in Southern Hill Area Key Laboratory of Sichuan Province, Chengdu, 14 Sichuan, China

15 Corresponding Author:

Qing Li, Rulin Wang

Wenjiang District, Chengdu, Sichuan, 611130, China

Email address: 10100@sicau.edu.cn; 398927566@qq.com

\section{Abstract}

Background. Citrus huanglongbing (HLB) is a destructive disease of citrus and a major threat to the citrus industry around the world. This disease accounts for substantial economic losses in China every year. Diaphorina citri Kuwayama is one of the major vectors by which citrus HLB is spread under natural conditions in China. Research is needed to identify the geographic distribution of $D$. citri and its major areas of occurrence and to formulate measures for early warning, monitoring and control of this pest and citrus HLB.

Methods. In this study, the ecological niche modelling software MaxEnt (maximum entropy model) was combined with ArcGIS (a geographic information system) to predict the potential geographic distribution of $D$. citri in China. Key environmental factors and the appropriate ranges of their values were also investigated. 
33 Results. Our results show that the training data provided a good forecast $\left(\mathrm{AUC}_{\text {mean }}=0.988\right)$. The highly suitable areas for $D$. citri in China are mainly concentrated to the south of the

Yangtze River, and the total area is $139.83 \times 10^{4} \mathrm{~km}^{2}$. The area of the moderately suitable areas is $27.71 \times 10^{4} \mathrm{~km}^{2}$, with a narrower distribution than that of the highly suitable area. The important environmental factors affecting the distribution of $D$. citri were Min Temperature of Coldest Month, Mean Temperature of Coldest Quarter, Precipitation of Wettest Quarter, Mean Temperature of Warmest Quarter, Precipitation of Warmest Quarter, Max Temperature of Warmest Month, and Temperature Seasonality. These results provide a valuable theoretical basis for risk assessments and control of $D$. citri.

42

43

44

45

46

47

48

49

Discussion. The predicted results showed that there were highly suitable areas for D. citri in Chongqing, Hubei, Anhui and Jiangsu. Therefore, the possibility exists for the further spread of D. citri in China in the future. Extreme temperature variables, especially the Min temperature of the coldest month, play an important role in the distribution of D. citri and are most closely related to the distribution of $D$. citri.

\section{Introduction}

Diaphorina citri Kuwayama (Hemiptera: Liviidae) is a major pest of Rutaceae plants, including Citrus reticulata Blanco, Citrus sinensis Osbeck, Citrus maxima Merr., and Murraya paniculata Jack (Miranda et al., 2017; Yao et al., 2018). Diaphorina citri mainly damages the new shoots of citrus plants. Adults occur on the leaves and buds, whereas nymphs cluster on shoots, buds and young leaves. The damaged shoots and buds wither, and the deformed leaves fall off easily, which seriously affects the growth of the plants. The white secretions from the nymphs affect photosynthesis in the branches and leaves (Yao et al., 2018). In addition to direct feeding damage, the greatest harm caused by $D$. citri is the transmission of the huanglongbing (HLB) pathogen (Manjunath et al., 2008; Fan et al., 2010). The pathogen can circulate, diffuse and proliferate in the body of $D$. citri and has evolved a set of mechanisms to evade the immune system of the pest. When infected $D$. citri feed on healthy plants, the pathogen can be introduced into the plant through the mouthparts of $D$. citri and establish, reproduce and expand in the plant (Luo et al., 2015; Song and Luo, 2017).

Citrus HLB is a worldwide citrus disease and is the most harmful and destructive disease in the citrus industry (Luo et al., 2017). As early as the middle of the 18th century, reports were made of citrus HLB in India. At present, citrus HLB occurs in more than 40 countries in Asia, Africa, North America and South America, which seriously threatens the development of the global citrus industry (Narouei-Khandan et al., 2016). The first report of the occurrence of citrus HLB in China was the 1920s, and the disease has spread rapidly in the southern citrus-producing areas of China. By the end of the 1970s, the disease occurred in Sichuan and Jiangxi except for Guangdong, Guangxi, Fujian, or Taiwan (Hu and Zhou, 2010). At present, 11 of the 19 provinces with citrus cultivation have been harmed by citrus HLB, and the affected area accounts for more than $80 \%$ of the total citrus cultivation area (Fan et al., 2009; Cheng et al., 2013). 
74

75

76

77

78

79

80

81

82

83

84

85

86

87

88

89

90

91

92

93

94

95

96

97

98

99

100

101

102

103

104

105

106

107

108

109

110

111

112

113

114

115

116

117

118

119

According to reports, citrus HLB occurred in Guangdong Province in 2016, with an area of $8 \times 10^{4} \mathrm{hm}^{2}$, resulting in losses of more than 1 billion dollars (Cheng et al., 2016).

Diaphorina citri was first recorded in Taiwan in 1907, and it is also believed to have originated in India (Kuwayama S, 1908; Husain and Nath, 1927). Worldwide, D. citri is currently distributed in Asia (China, India, Sri Lanka, Malaysia, Indonesia, Philippines, Bangladesh, Thailand, Iran, Bhutan and Afghanistan) (Bové, 2006; Sule et al., 2012; Lashkari et al., 2014; Wang et al., 2017; Wang et al., 2018a), North America (USA, Mexico, Honduras, Bahamas, Cayman Islands and Jamaica) (Halbert and Nunez, 2004; López-Collado et al., 2013; Milosavljević et al., 2018), South America (Brazil, Colombia, Ecuador and Paraguay) (Cornejo and Chica, 2014; Narouei-Khandan et al., 2016), Africa (Kenya, Mauritius and Tanzania) (Shimwela et al., 2016; Rwomushana et al., 2017) and Australia (Aurambout et al., 2009). In China, Diaphorina citri is mainly distributed in Guangdong, Guangxi, Taiwan, Macao, Hong Kong, Fujian, Zhejiang, Jiangxi, Hunan, Guizhou, Yunnan, Sichuan and Hainan (Wang et al., 2016).

Meteorological factors are important environmental factors affecting the distribution, occurrence and development of pests and diseases. Global warming is a long-term increase in the average temperature of the Earth's climate system, an aspect of climate change shown by temperature measurements and multiple effects of warming (Craufurd and Wheeler, 2009). Under the background of climate warming, the suitable areas for pests and diseases are increasing, which leads to the expansion of their geographical distributions (Monteith, 2000). Temperature is one of the main factors restricting the distribution of pests on Earth (Zhang et al., 2012). Climate warming increases the chance of insects restricted by low temperatures to spread to high altitudes ( $\mathrm{Hu}$ et al., 2015). In recent years, with the increase of winter temperatures, the population of $D$. citri has expanded significantly, and its geographical distribution has spread northward each year, which has aggravated the speed of spread and damage scope of citrus HLB (Chen et al., 2016; Wang et al., 2016). Therefore, research on the influence of climate factors on the distribution of $D$. citri is important.

MaxEnt is a habitat suitability model based on the niche principle that uses species distribution data and environmental data to analyse the distribution state of species at maximum entropy (Jayakumar et al., 2015). Compared with other niche models, the model has higher prediction accuracy and can obtain satisfactory results with relatively few distribution points (Elith et al., 2006; Petitpierre et al., 2012; Zhang et al., 2016). The MaxEnt model has been widely used by scholars in China and abroad. Kumar et al., (2014) used MaxEnt to predict the invasion potential of the exotic pest Phenacoccus solenopsis Tinsley (Hemiptera: Coccoidea: Pseudococcidae) in India. Penado et al., (2016) used MaxEnt to identify climatically suitable areas for bumblebees in under-sampled parts of the Iberian Peninsula. López-Martínez et al., (2016) studied the environmental suitability for Agrilus auroguttatus Schaeffer (Coleoptera: Buprestidae) in Mexico using MaxEnt. Lozier and Mills (2011) used the correlative niche modelling method MaxEnt to predict the geographic distribution of Epiphyas postvittana Walker (Lepidoptera: Tortricidae) in its native range and globally and tested model projections using known invasion data.

Recent research on D. citri has mainly focused on biological characteristics (Ruan et al., 2012), ecological characteristics (Onagbola et al., 2008), comprehensive control measures (Manjunath et al., 2008; Jia et al., 2015; Wang et al., 2018b), the host selection mechanism and the transmission mechanism of HLB (Inoue et al., 2009; Hijaz et al., 2016; Arp et al., 2017; Killiny et al., 2017; Yu and Killiny, 2018); research regarding prediction of its geographical 
120 distribution in China is relatively rare. To provide a theoretical basis for such prediction, risk

121

122

123

124

125

126

127

128

129

130

131

132

133

134

135

136

137

138

139

140

141

142

143

144

145

146

147

148

149

150

151

152

153

154

155

156

157

158

159

160

161

162

163

164

assessments and the effective control of $D$. citri, the MaxEnt model was used to map the potential distribution of D. citri in China under current climate conditions, and the relationship between the distribution of $D$. citri and environmental factors was elaborated in this paper.

\section{Materials \& Methods}

\section{Environmental variables and species data}

To obtain the occurrence records of $D$. citri in China, we accessed two online databases, the European and Mediterranean Plant Protection Organization database $(E P P O, 2018)$ and the Global Biodiversity Information Facility database $(G B I F, 2018)$, and consulted many published articles (Halbert and Manjunanth, 2004; Yang et al., 2006; Wang et al., 2017a; Jiang et al., 2018). According to the method described by Jiang et al. (2018) to filter distribution records, we used Google Earth (Collette and Pither, 2015) to proofread the latitude and longitude. In strict accordance with the requirements of MaxEnt, duplicate records, fuzzy records and neighbouring records were removed. Finally, 135 valid records were retained for constructing the models.

In this study, to analyse the climatic suitability regionalization of $D$. citri in China, we chose climatic factors and altitude factors as the initial environmental variables. Data on climate variables and altitude were downloaded from the official WorldClim website (Fick and Hijmans, 2017) (Table S1), which provides the average data from 1950 to 2000. MaxEnt is a mathematical model based on the principle of climate similarity; it is used to explore the correlation between geographical distribution and environmental variables (Elith et al., 2006). The choice of environmental variables is the key to determining the accuracy of the simulation. Multiple collinearity may exist among the environmental variables, which affects the model's evaluation of response relationships and contribution rates, which in turn affects the accuracy of the simulation (Zhang and Liu, 2017). MaxEnt computes the contribution of predictor variables to model the potential occurrence of a species. In this study, we refer to Zhang and Liu's (2017) method to screen environmental variables. The screening procedure is as follows: 1 . Establishment of an initial model to calculate the contribution of the variables to the model (Table 1). 2. Use of ArcGIS to extract the attribute values for 20 variables at each of the 135 presence records and SPSS to calculate the Pearson correlation coefficients between any two variables. 3. If a correlation coefficient is greater than 0.8 , the most relevant variable is retained according to the percent contribution of the variable in the initial model, and the other variable is excluded. 4. The remaining variables are sorted according to the percent contribution, and the variables with a percent contribution greater than 1.0 are retained. Through the above procedure and referring to the biological characteristics of $D$. citri, the screening of environmental variables was completed.

\section{Modelling method and statistical analysis}

MaxEnt builds a model by means of a machine-learning algorithm to predict the suitability for the occurrence of a given species in a spatial dimension. MaxEnt software (version 3.4.1), which is now open source, was downloaded from the website of the American Museum of Natural History (Phillips et al., 2019); this software has excellent predictive performance for pests and diseases (Jarnevich and Young, 2015).

The specific operational steps for MaxEnt are described herein. First, we imported the occurrence points for D. citri and data for 20 variables into the MaxEnt software to create the

Peer] reviewing PDF | (2019:01:34434:2:1:NEW 1 Jun 2019) 
165

166

167

168

169

170

171

172

173

174

175

176

177

178

179

180

181

182

183

184

185

186

187

188

189

190

191

192

193

194

195

196

197

198

199

200

201

202

203

204

205

206

207

208

initial model. In the initial model, the 'Random test percentage' was set as 25 , and 'Make pictures of predictions' and 'Do jackknife to measure variable importance' were chosen; the remaining model values were set to default values. Then, we evaluated the percent contributions of the environmental variables to select variables for modelling. Finally, the occurrence points and the selected environmental variables were uploaded to MaxEnt to simulate the distribution of D. citri in China. In the final model, "Random seed" was chosen, and 10 replicate models were run. We selected the best model with the highest AUC (Area unde the receiver operating characteristic curve) value. The remaining model settings were set to the same as those in the initial model (Kumar and Stohlgren, 2009; Zhu et al., 2018).

Response curves indicate the relationship between the probability of $D$. citri occurrence and environmental variables was computed by MaxEnt. We use the response curves for each variable to avoid the influence of correlation between variables. Generally, the ecological factor value suitable for the presence of $D$. citri is generally believed to be the value when the probability of the presence of $D$. citri is greater than 0.33. ArcGIS software was used to superimpose the index distribution map with China's administrative division map to obtain the suitability regionalization map for D. citri. We reclassified the distribution threshold and divided the suitable area into 4 categories, displaying them in different colour according to the method described by Wang et al., $(2018 c)$.

Analysis of the receiver operating characteristic (ROC) curve is an effective method for evaluating the accuracy of the species distribution model. The method uses the area under the curve (AUC) as the index to measure the model accuracy. The theoretical value range of the AUC is 0.5 1; AUC values closer to 1 indicate a higher prediction accuracy of the model. The evaluation criteria are simulation failure (fail), $0.5 \leq \mathrm{AUC}<0.6$; poor simulation results (poor), $0.6 \leq \mathrm{AUC}<0.7$; generally fair simulation results (fair), $0.7 \leq \mathrm{AUC}<0.8$; good simulation results (good), $0.8 \leq \mathrm{AUC}<0.9$; and excellent simulation results (excellent), $0.9 \leq \mathrm{AUC}<1$ (Wang et al., 2007).

\section{Results}

\section{Model performance}

Figure 1-a shows the ROC curve of the initial model. The AUC values of the training data and the test data are 0.966 and 0.956 , respectively. According to the evaluation criteria described in 'Materials \& Methods', the accuracy of the initial model is "excellent". Figure 1-b shows the ROC curve of the final model. The results show that the mean AUC value of the 10 replicates was 0.988 .

\section{Selection of the environmental variables}

Pearson correlation coefficients between the 20 environmental variables are shown in Table S2. The correlation coefficient between Altitude and Precipitation of Warmest Quarter, Annual Mean Temperature and Temperature Annual Range, Annual Mean Temperature and Mean Temperature of Wettest Quarter, Mean Temperature of Wettest Quarter and Precipitation of Driest Month, Mean Temperature of Driest Quarter and Mean Temperature of Coldest Quarter were greater than 0.8, and Altitude, Temperature Annual Range, Mean Temperature of Wettest Quarter and Mean Temperature of Driest Quarter were excluded according to the screening procedure. The results show that the percent contributions of Mean Temperature of Coldest

Peer) reviewing PDF | (2019:01:34434:2:1:NEW 1 Jun 2019) 
209 Quarter, Precipitation of Warmest Quarter, Temperature Seasonality, Precipitation of Driest

210 Month, Precipitation Seasonality, Min Temperature of Coldest Month, Precipitation of Wettest

211 Quarter, and Mean Temperature of Warmest Quarter were higher than 1.0\%, and the cumulative

212 sum was $97.2 \%$, which was significantly higher than that of the other variables (Table 1).

213 Previous biological studies have shown that extreme temperature variables play an important role

214 in the distribution of D. citri (López-Collado et al., 2013). Therefore, the Max Temperature of

215 Warmest Month was also selected for the final model. Finally, nine variables were selected:

216 Mean Temperature of Coldest Quarter, Precipitation of Warmest Quarter, Temperature

217 Seasonality, Precipitation of Driest Month, Precipitation Seasonality, Min Temperature of

218 Coldest Month, Precipitation of Wettest Quarter, Mean Temperature of Warmest Quarter and

219 Max Temperature of Warmest Month. On this basis, the final model for the distribution of $D$.

220 citri in China was established, and the accuracy of the simulation results was evaluated.

221

222

223

224

225

226

227

228

229

230

231

232

233

234

235

236

237

238

239

240

241

242

243

244

245

246

247

248

249

250

251

252

253

254

255

\section{The potential distribution of $D$. citri in China}

The selected environmental variables were combined in the MaxEnt model to obtain a suitable index distribution map of $D$. citri in China (Fig. 2). The results showed that the highly suitable areas for D. citri in China are mainly concentrated to the south of the Yangtze River, including Guangxi, Guangdong, Hunan, Jiangxi, Fujian, most of Guizhou, north-eastern Hainan, western Taiwan, southern and central Zhejiang, northern Yunnan, east-central Sichuan, most of Chongqing, and Hong Kong (Fig. 2). The total area of the highly suitable area in China is $139.83 \times 10^{4} \mathrm{~km}^{2}$, which occupies $14.52 \%$ of the area of the national territory. The moderately suitable areas are mainly distributed in southern Yunnan, southern Hubei, southern Hunan, central Hainan, southern Taiwan, southern Jiangsu and central Zhejiang. The total area of the moderately suitable area is $27.71 \times 10^{4} \mathrm{~km}^{2}$, with a narrower distribution than the most suitable area. The total suitable area (the highly suitable area and the moderately suitable area) is $167.54 \times 10^{4} \mathrm{~km}^{2}$, accounting for $17 \%$ of China's total area.

\section{Key environmental variables in the model}

The results of the jackknife test can reflect the importance of the environmental variables to the model by calculating the training gains when using 'with only variable', 'without variable' and 'with all variables' for the simulation. Figure 3 is the result of the analysis of the importance of environmental variables to the distribution of $D$. citri according to the jackknife test. It can be seen from the figure that the Min Temperature of Coldest Month is the most important environmental variable affecting the distribution of $D$. citri in China, and its training gain exceeds 1.7. Mean Temperature of Coldest Quarter, Precipitation of Wettest Quarter and Mean Temperature of Warmest Quarter were also important environmental variables, and their individual training gains were greater than 1.6. The order of importance of the nine environmental variables is Min Temperature of Coldest Month $>$ Mean Temperature of Coldest Quarter $>$ Precipitation of Wettest Quarter $>$ Mean Temperature of Warmest Quarter $>$ Precipitation of Warmest quarter $>$ Max Temperature of Warmest Month $>$ Temperature Seasonality $>$ Precipitation of Driest Month $>$ Precipitation Seasonality.

\section{Environmental variables affecting the occurrence of $\boldsymbol{D}$. citri}

As shown in Fig. 4, when the Min Temperature of Coldest Month is below $-3.58^{\circ} \mathrm{C}$, the probability of $D$. citri presence is less than 0.33 . With the increase in Min Temperature of Coldest Month, the probability increased rapidly and reached its highest point at $23.4{ }^{\circ} \mathrm{C}$. The change in the response curve of the Mean Temperature of Coldest Quarter is different from that 
256

257

258

259

260

261

262

263

264

265

266

267

268

269

270

271

\section{Discussion}

273

274

275

276

277

278

279

280

281

282

283

284

285

286

287

288

289

290

291

292

293

294

295

296

297

for the Min Temperature of Coldest Month, and the suitable range of the Mean Temperature of Coldest Quarter is $6.03-17.88^{\circ} \mathrm{C}$. When the temperature is lower than $6.03{ }^{\circ} \mathrm{C}$ or higher than $17.88^{\circ} \mathrm{C}$, the probability of $D$. citri occurrence is lower than 0.33 and reaches its highest value at $10.26^{\circ} \mathrm{C}$. When the precipitation of wettest quarter is below $548.66 \mathrm{~mm}$, the probability of $D$. citri presence is lower than 0.33 . With an increase in precipitation of wettest quarter, the probability of the presence of $D$. citri increased rapidly and reached its highest value at 619.02 $\mathrm{mm}$. After that, the probability of $D$. citri presence slowly decreases. When precipitation of wettest quarter reaches approximately $1189.75 \mathrm{~mm}$, the probability of the presence of $D$. citri falls below 0.33 . Therefore, the suitable range of precipitation of wettest quarter for $D$. citri is $562.89-1189.75 \mathrm{~mm}$.

The trends of presence probability in association with the variables (Mean Temperature of Warmest Quarter, Precipitation of warmest quarter, Max Temperature of Warmest Month, Temperature Seasonality) are similar to that of Mean Temperature of Coldest Quarter, and the suitable ranges were $24.46-34.27^{\circ} \mathrm{C}, 503.73-1533.58 \mathrm{~mm}, 28.6-40.91{ }^{\circ} \mathrm{C}$, and $56.83-818.03$, respectively (Fig. 4).

\section{Evaluation of the MaxEnt model}

Commonly used model evaluation indicators include overall accuracy, sensitivity, specificity, kappa, and true skill statistic (TSS) (Allouche et al., 2006; Wang et al., 2007). The ROC curve is not affected by the threshold and is considered to be one of the best evaluation indicators at present. MaxEnt software can directly draw the ROC curve and calculate the AUC value of the model, which is convenient for judging the predictive effect of the model. Therefore, ROC curves are widely used in the evaluation of MaxEnt models. For example, Wang et al. (2017b) used ROC curves to evaluate the predictive effect of the MaxEnt model in terms of suitable habitats for the Colorado potato beetle at a global scale; Han et al. (2015) used ROC curves to determine the accuracy of niche models in predicting suitable habitats for Bursaphelenchus xylophilus Steiner \& Bohrer (Tylenchida Thorne: Sphelenchoidae) in China. Therefore, the ROC curve is used to evaluate the predictive accuracy of the MaxEnt model. The stability of the model is verified by 10 repeated AUC values. The average AUC value of 10 replicated runs of the model was 0.988 , which indicates that the simulation effect was "excellent" and proves that the model can be used to simulate the potential distribution of $D$. citri in China.

\section{Predicting the distribution of $D$. citri in China}

We used the "extraction" tool in ArcGIS software to identify suitable areas for D. citri in China. According to previous study results, the suitable areas were divided into four grades: highly suitable areas, moderately suitable areas, low-suitability areas and unsuitable areas, and the suitable areas of each grade were calculated. The results showed that the highly suitable areas for $D$. citri in China are mainly concentrated to the south of the Yangtze River, and the total area of the highly suitable area in China is $139.83 \times 10^{4} \mathrm{~km}^{2}$. The moderately suitable areas are distributed to the north of the highly suitable areas, and the area is $27.71 \times 10^{4} \mathrm{~km}^{2}$. The total suitable area (the highly suitable area and the moderately suitable area) is $167.54 \times 10^{4} \mathrm{~km}^{2}$ and

Peer) reviewing PDF | (2019:01:34434:2:1:NEW 1 Jun 2019) 
298

299

300

301

302

303

304

305

306

307

308

309

310

311

312

313

314

315

316

317

318

319

320

321

322

323

324

325

326

327

328

329

330

331

332

333

334

335

336

337

338

339

340

341

342

343 accounts for $17 \%$ of China's total area. Wang et al. (2015) applied CLIMEX to predict the potential distribution of $D$. citri in China, and the results were basically consistent with our predictions, with our predicted habitat being more to the north. This difference may have occurred because of the use of different prediction models, species distribution data and environmental variables.

The distribution of $D$. citri in China was investigated by the National Agricultural Technology Extension Service Center in 2014. The results showed that D. citri occurred in Zhejiang, Jiangxi, Hunan, Sichuan, Guizhou, Yunnan, Guangxi, Guangdong and Hainan but not in Chongqing and Hubei (Wang et al., 2016). The predicted results showed that there were highly suitable areas for D. citri in Chongqing, Hubei, Anhui and Jiangsu. Chongqing and Hubei are located in the core area of the citrus industry belt along the middle and upper reaches of the Yangtze River, which is one of the most suitable areas for citrus cultivation in China (Xiang et al., 2014). By the end of 2017, the cultivated areas of citrus in Hubei and Chongqing were $21.33 \times 10^{4} \mathrm{hm}^{2}$ and $41.28 \times 10^{4} \mathrm{hm}^{2}$, respectively (Ding et al., 2017). Therefore, the above two provinces had suitable conditions for the colonization of $D$. citri. Southern Chongqing is adjacent to Sichuan, Chongqing and Hunan, and D. citri has been found in these provinces in recent years. Geographically, southern Chongqing is adjacent to Sichuan, Yunnan and Hunan, while southern Hubei is adjacent to Hunan and Jiangxi. Diaphorina citri has been found in Sichuan, Yuan, Hunan and Jiangxi in recent years. Since the 1980s, the occurrence boundary of D. citri has been moving northward (Chen et al., 2016). Therefore, the possibility of introduction and colonization of $D$. citri in Chongqing and Hubei is very high, and inspection and quarantine work should be strengthened to prevent the introduction of $D$. citri to these regions. Because the climate is not suitable for citrus growth, there is almost no citrus planting in Anhui and Jiangsu, and the suitable areas for $D$. citri in Anhui and Jiangsu are very small $(W u, 2018)$. Therefore, we speculate that the probability of occurrence of $D$. citri in this area is very low and will not cause serious economic losses.

Hosts of D. citri include Citrus reticulata Blanco, Citrus sinensis Osbeck, Citrus maxima Merr., Murraya paniculata Jack, Murraya exotica L., and Clausena lansium (Lour.) Skeels. These host plants have different effects on the development, reproduction and survival of $D$. citri. Ren et al. (2018) investigated the life history of five different host plants. The results showed that the adult longevity of $D$. citri was significantly affected by the host plant and was highest on $C$. maxima and shortest on $C$. lansium, while the survivorship of larvae was highest (58.10\%) on C. maxima and lowest on C. lansium (46.04\%). Tsai and Liu (2000) showed that the survival rate of $D$. citri was highest on grapefruit and lowest on lime. Chen et al. (2011) measured the feeding preferences of $D$. citri among 13 citrus varieties and found that the number of $D$. citri on the shoots of Newhall navel orange and Fuju was significantly higher than that on the other 11 citrus varieties. These previous studies have shown the importance of host identity for the distribution of $D$. citri, and the analysis of the dependence of $D$. citri on hosts can help to improve the accuracy of predictions. In this paper, we did not simulate the suitable habitats of various hosts of $D$. citri in China, but we can confirm that the main citrus-producing areas cover the suitable habitats of $D$. citri according to the relevant literature. Therefore, the results of this study are still highly reliable.

\section{Environmental variables affecting the geographical distribution of $\boldsymbol{D}$. citri}

The occurrence, growth, and spread of plant diseases and insect pests depend not only on the biological characteristics of the disease and pests but also on the host plants, farming 
344 systems, management levels and environmental conditions. Meteorological variables are 345 extremely important environmental factors. Under other conditions that are relatively consistent, 346 meteorological factors will become a decisive factor affecting the epidemic or large-scale 347 outbreak of pests and diseases (Monteith, 2000; Qin et al., 2017). Studies have shown that 348 abiotic factors such as temperature, humidity and atmospheric pressure can affect the distribution 349 of D. citri (Martini and Stelinski, 2017), and variables related to temperature are more critical in predictions (López-Collado et al., 2013). Therefore, we focus on the effect of temperature on the geographical distribution of citrus as follows.

López-Collado et al. (2013) found that the minimum temperature of the coldest month seemed to be the most important variable affecting the distribution of $D$. citri. In this paper, the importance of environmental variables was tested with the jackknife method. The results showed that the Min Temperature of Coldest Month was the most important variable, indicating that it is most closely related to the distribution of $D$. citri, which is consistent with the result found by López-Collado et al. The response curve showed that the probability of the presence of $D$. citri was very low when the Min Temperature of Coldest Month was below $-3.58^{\circ} \mathrm{C}$, which indicates that extremely low temperatures limit the distribution of D. citri. In India, Atwal et al., (1970) found that extremely low temperatures were not conducive to the development of the population of $D$. citri. Low temperatures below $0^{\circ} \mathrm{C}$ played an important role in suppressing the population of D. citri, and Yang et al., (2006) found the same rule in China. Bai et al., (2008) found that the annual minimum average temperature was the main factor limiting the geographical distribution of D. citri. Hall et al., (2011) noted that the low temperature in winter was the main factor limiting the population growth, geographical distribution and potential transmission of $D$. citri. These results are consistent with the results of this study.

Studies have shown that the damage caused by high temperatures to insects is irreversible and has a certain accumulative effect. Exposure to high temperatures for a long time will lead to a significant decrease in the water content of insects and even their death (Zhong, 2010; Zhang et al., 2014). Investigating the effects of high temperature on the mortality and activity behaviour of $D$. citri demonstrated that a high temperature above $40^{\circ} \mathrm{C}$ leads to a decrease in the survival time of the D. citri population (Kuang et al., 2017). Hall et al., (2011) estimated temperature thresholds for the oviposition of D. citri and found that the lower and upper thresholds for oviposition were 16 and $41.6^{\circ} \mathrm{C}$, respectively. The results of this study indicate that the Max Temperature of Warmest Month is also a key variable affecting the distribution of D. citri and that the suitable range is $28.6-40.91^{\circ} \mathrm{C}$, which is consistent with the above conclusions. NaroueiKhandan et al. (2016) showed that the presence of $D$. citri was limited when the mean temperature in the warmest season was higher than $33^{\circ} \mathrm{C}$. The response curve of the Mean Temperature of Warmest Quarter showed that the upper limit temperature for the presence of $D$. citri was $34.27^{\circ} \mathrm{C}$. This result may have occurred because the larvae and eggs of $D$. citri are more sensitive to high temperatures than adults (Liu and Tsai, 2000). In this paper, the response curve shows the effect of a single environmental variable on species distribution, but the growth and distribution of $D$. citri depend on the comprehensive effect of various environmental factors. Therefore, this conclusion cannot fully explain the relationship between $D$. citri and the environmental variables but can be used as a theoretical reference to evaluate the relationship between them. 
388

389

390

391

392

393

394

395

396

397

398

399

400

401

402

403

404

405

406

\section{Figure Legends}

408 Figure 1:

409

410

411

412

413

414

415

416

417

418

419

420

421

422

423

424

425

426

427

428

\section{Conclusions} control of D. citri.

\section{Figure 2:} Figure 3:

Figure 4:

\section{References} 2524. 1232.

Based on MaxEnt software and certain environmental data, this study predicts the geographical distribution of $D$. citri in China and aims to provide a scientific reference for the

In this study, the occurrence data of $D$. citri were mainly obtained from EPPO, GBIF, and the literature, and the usable data were much fewer than the available data. The longitude and latitude of some distribution points are obtained by using positioning software, so there is inevitably some geographic error. The basic niche refers to the largest niche that is occupied by a species under the most ideal living conditions. The niche model only analyses the influence of abiotic factors on species distributions, suggesting that the niche predicted by the model is wider than the actual niche occupied by $D$. citri. Therefore, the results of this study have certain limitations and shortcomings.

Studies have shown that in the past 20 years, with increasing global warming, the growth and distribution patterns of the species have changed significantly (Ma et al., 2013; Zhao et al., 2014; Pacifici, et al., 2017). The lack of climate data in the past 20 years may lead to a deviation in the conclusions from the actual situation. Therefore, to ensure more reliable prediction results, more comprehensive and accurate distribution data for $D$. citri should be used, and the corresponding missing climate data should be supplemented in the next step.

ROC curve and AUC values for the initial model (a) and the final model (b).

Probability (P) of the potential distribution of $D$. citri in China based on the MaxEnt model.

Jackknife test for variable importance in the $D$. citri suitability distribution: values shown are averages over 10 replicate runs.

Response curves of environmental variables in the MaxEnt models: (A) BIO6; (B) BIO11; . (C) BIO16; (D) BIO18; (E) BIO5; (F) BIO4; (G) BIO10.

Arp AP, Martini X, Pelz-Stelinski KS. 2017. Innate immune system capabilities of the Asian citrus psyllid, Diaphorina citri. Journal of invertebrate pathology 148: 94-101.

Aurambout JP, Finlay KJ, Luck J, Beattie GAC. 2009. A concept model to estimate the potential distribution of the Asiatic citrus psyllid (Diaphorina citri Kuwayama) in Australia under climate change-a means for assessing biosecurity risk. Ecological Modelling 220(19): 2512-

Allouche O, Tsoar A, Kadmon R. 2006. Assessing the accuracy of species distribution models: prevalence, kappa and the true skill statistic (TSS). Journal of Applied Ecology 43(6): 1223- 
429

430

431

432

433

434

435

436

437

438

439

440

441

442

443

444

445

446

447

448

449

450

451

452

453

454

455

456

457

458

459

460

461

462

463

464

465

466

467

468

469

470

471

472
Atwal AS, Chaudhary JP, Ramzan M. 1970. Studies on the development and field population of citrus psylla, Diaphorina citri kuwayama (Psyllidae: Homoptera). Journal of ResearchPunjab Agricultural University 7: 333-338.

Bové JM. 2006. Huanglongbing: a destructive, newly-emerging, century-old disease of citrus. Journal of Plant Pathology 88(1): 7-37.

Bai XJ, Deng CL, Lu BG, Zhao XL, Deng GZ, Li GG, Chen CW, Wu RC, Tang Y, Chen GP, Fu HM, Mo JS. 2008. Investigation on the cold tolerance of Asian citrus psyllid. South China Fruits 37(6): 22-24.

Cheng CZ, Zeng JW, Zhong Y, Yan HX, Jiang B, Zhong GY. 2013. Research progress on citrus huanglongbing disease. Acta Horticulturae Sinica 40: 1656-1668.

Cheng BP, Zhao HW, Peng AT, Song XB, Ling JF, Chen X. 2016. Occurrence of huanglongbing disease and its vector-citrus psylla in citrus orchard of Guangdong province. Plant Protection 42(1): 189-192.

Cornejo JF, Chica EJ. 2014. First record of Diaphorina citri (Hemiptera: Psyllidae) in ecuador infesting urban citrus and orange jasmine trees. Journal of Insect Science 14(1): 298-298.

Craufurd PQ, Wheeler TR. 2009. Climate change and the flowering time of annual crops. Journal of Experimental Botany, 60(9): 2529-2539.

Chen LF, Xu ZX, Wang JG. 2016. Research progress on Diaphorina citri. Guizhou Agricultural Sciences 44(6): 42-47.

Collette LKD, Pither J. 2015. Modeling the potential North American distribution of Russian olive, an invader of riparian ecosystems. Plant Ecology 216(10): 1371-1383.

Chen JL, Ruan CQ, Liu B, Fan GC, Duan YP, Hall D. 2011. Diaphorina citri's gost preference on thirteen citrus varieties. Fujian Journal of Aaricultural Sciences 26(2):280-283.

Ding WY, Yuan B, Zhou YH. 2017. Analysis on growth pattern and trend of fruit industry in China: take citrus for example. World Agriculture 12: 148-155.

Elith J, Graham CH, Anderson RP, Dudík M, Ferrier S, Guisan A, Hijmans RJ, Huettmann F, Leathwick JR, Lehmann A, Jin L, Lohmann LG, Loiselle BA, Manion G, Moritz C, Nakamura M, Nakazawa Y, McC. Overton J, Peterson AT, Phillips SJ. 2006. Novel methods improve prediction of species' distributions from occurrence data. Ecography 29(2): 129-151.

EPPO. 2018. EPPO global database. Available at https://gd.eppo.int (Accessed on 2018).

Fan J, Chen C, Brlansky RH, Gmitter FG, Li ZG. 2010. Changes in carbohydrate metabolism in citrus sinensis infected with 'Candidatus Liberibacter asiaticus'. Plant Pathology 59(6): 1037-1043.

Fan GC, Liu B, Wu RJ, Li T, Cai ZJ, Ke C. 2009. Thirty years of research on citrus huanglongbing in China. Fujian Journal of Agricultural Sciences 24(2): 183-190.

GBIF. 2018. Global Biodiversity Information Facility database. Available at https://www.gbif.org (Accessed on 2018).

Fick SE, Hijmans RJ . 2017. WorldClim 2: new 1-km spatial resolution climate surfaces for global land areas. International Journal of Climatology 37(12): 4302-4315.

$\mathrm{Hu}$ WZ, Zhou CY. 2010. Advances in the pathogen of citrus huanglongbing. Plant Protection 36(3): 30-33.

Husain MA, Nath LD. 1927. The citrus psylla (Diaphorina citri, Kuw.) [Psyllidae: Homoptera] Memoris of the department of agriculture. Entomological Series 10(2): 1-27.

PeerJ reviewing PDF | (2019:01:34434:2:1:NEW 1 Jun 2019) 
473

474

475

476

477

478

479

480

481

482

483

484

485

486

487

488

489

490

491

492

493

494

495

496

497

498

499

500

501

502

503

504

505

506

507

508

509

510

511

512

513

514

515

516

517

Halbert SE, Manjunanth KL. 2004. Asian citrus psyllids (Sternorrhycha: Psyllidae) and greening disease of citrus: A literature review and assessment of risk in Florida. Florida Entomologist 87: 330-353.

Hu CX, Hou ML, Wei GS, Shi BX, Huang JL. 2015. Potential overwintering boundary and voltinism changes in the brown planthopper, Nilaparvata lugens, in China in response to global warming. Climatic Change 132(2):337-352.

Hijaz F, Lu ZJ, Killiny N. 2016. Effect of host-plant and infection with 'Candidatus Liberibacter asiaticus' on honeydew chemical composition of the Asian citrus psyllid, Diaphorina citri. Entomologia Experimentalis et Applicata 158(1): 34-43.

Halbert SE, Manjunanth KL. 2004. Asian citrus psyllids (Sternorrhycha: Psyllidae) and greening disease of citrus: A literature review and assessment of risk in Florida. Florida Entomologist 87: 330-353.

Han YY, Wang Y, Xiang Y, Ye JR. 2015. Prediction of potential distribution of Bursaphelenchus xylophilus in China based on Maxent ecological niche model. Journal of Nanjing Forestry University 1: 6-10.

Hall DG, Wenninger EJ, Hentz MG. 2011. Temperature studies with the Asian citrus psyllid, Diaphorina citri: cold hardiness and temperature thresholds for oviposition. Journal of Insect Science 11(1): 83.

Inoue H, Ohnishi J, Ito T, Tomimura K, Miyata S, Iwanami T, Ashihara W. 2009. Enhanced proliferation and efficient transmission of Candidatus Liberibacter asiaticus by adult Diaphorina citri after acquisition feeding in the nymphal stage. Annals of Applied Biology 155(1): 29-36.

Jayakumar S, Remyaa K, Ramachandrana A. 2015. Predicting the current and future suitable habitat distribution of Myristica dactyloides Gaertn. using MaxEnt model in the Eastern Ghats, India. Ecological Engineering 82(9): 184-188.

Jia ZC, Zheng JQ, Huang YJ, Zhou HP, Ehsani, R. 2015. Review and prospect of thermotherapy for citrus huanglongbing. Transactions of the Chinese Society of Agricultural Engineering 31(23): 1-9.

Jiang HY, Wu FN, Wang YJ, Tao L, Wang JF, Liu Z, Cen YJ., 2018. Research advances on the origin, distribution and dispersal of the Asian citrus psyllid (Diaphorina citri Kuwayama). Journal of Environmental Entomology 40(5):1014-1020.

Jarnevich CS, Young N. 2015. Using the MAXENT program for species distribution modelling to assess invasion risk. Pest Risk Modelling and Mapping for Invasive Alien Species 2015: 65-81.

Kuwayama S. 1908. Die psylliden Japans. Transactions of the sapporo natural history society, 2: 149-189.

Kumar S, Graham J, West AM, Evangelista PH. 2014. Using district-level occurrences in MaxEnt for predicting the invasion potential of an exotic insect pest in India. Computers and Electronics in Agriculture 103: 55-62.

Killiny N, Hijaz F, El-Shesheny I, Alfaress S, Jones S E, Rogers M E. 2017. Metabolomic analyses of the haemolymph of the Asian citrus psyllid Diaphorina citri, the vector of huanglongbing. Physiological Entomology 42(2): 134-145.

Kumar S, Stohlgren TJ. 2009. Maxent modeling for predicting suitable habitat for threatened and endangered tree Canacomyrica monticola in New Caledonia. Journal of Ecology and Natural Environment 1(4): 94-98. 
518 Kuang F, Lin MQ, Yuan SX, Luo YQ, Zhang N, Lu ZJ, Hu W. 2017. Effects of high temperature 519 on mortality and activity behavior of Diaphorina citri kuwayama. Journal of Southern $520 \quad$ Agriculture 48(9): 1600-1604.

521

522

523

524

525

526

527

528

529

530

531

532

533

534

535

536

537

538

539

540

541

542

543

544

545

546

547

548

549

550

551

552

553

554

555

556

557

558

559

560

561

562

563

Luo XZ, Yen AL, Powell KS, Wu FN, Wang YJ, Zeng LX, Yang YZ, Cen YJ. 2015. Feeding behavior of Diaphorina citri (Hemiptera: Liviidae) and its acquisition of 'Candidatus liberibacter asiaticus', on huanglongbing-infected citrus reticulata leaves of several maturity stages. Florida Entomologist, 98: 186-192.

Luo L, Gao SJ, Ge YQ, Luo YQ. 2017. Transmission dynamics of a huanglongbing model with cross protection. Advances in Difference Equations 2017: 355.

Lashkari M, Manzari S, Sahragard A, Malagnini V, Boykin LM, Hosseini R. 2014. Global genetic variation in the Asian citrus psyllid, Diaphorina citri (Hemiptera: Liviidae) and the endosymbiont wolbachia: links between Iran and the USA detected. Pest Management Science 70(7): 1033-1040.

López-Collado J, López-Arroyo JI, Robles-García PL, Márquez-Santos M. 2013. Geographic distribution of habitat, development, and population growth rates of the Asian citrus psyllid, Diaphorina citri, in Mexico. Journal of Insect Science 13(1):114.

López-Martínez V, Sánchez-Martínez G, Jiménez-García D, B. Pérez-De la O N. 2016. Environmental suitability for Agrilus auroguttatus (Coleoptera: Buprestidae) in mexico using maxent and database records of four quercus (fagaceae) species. Agricultural and Forest Entomology 18(4): 409-418.

Lozier JD, Mills NJ. 2011. Predicting the potential invasive range of light brown apple moth (Epiphyas postvittana) using biologically informed and correlative species distribution models. Biological Invasions 13(10): 2409-2421.

Liu YH, Tsai JH. 2000. Effects of temperature on biology and life table parameters of the Asian citrus psyllid, Diaphorina citri Kuwayama (Homoptera: Psyllidae). Annals of Applied Biology 137(3): 201-206.

Miranda MP, Dos Santos FL, Bassanezi RB, Montesino LH, Barbosa JC, Sétamou M. 2017. Monitoring methods for Diaphorina citri Kuwayama (Hemiptera: Liviidae) on citrus groves with different insecticide application programmes. Journal of Applied Entomology 142(1-2): 89-96.

Manjunath KL, Halbert SE, Ramadugu C, Webb S, Lee RF. 2008. Detection of 'Candidatus Liberibacter asiaticus' in Diaphorina citri and its importance in the management of citrus huanglongbing in Florida. Phytopathology 98(4): 387-396.

Milosavljević I, Amrich R, Strode V, Hoddle MS. 2018. Modeling the phenology of Asian citrus psyllid (Hemiptera: Liviidae) in urban southern california: effects of environment, habitat, and natural enemies. Environmental entomology 47(2): 233-243.

Monteith JL. 2000. Agricultural meteorology: evolution and application. Agricultural and Forest Meteorology 103(1): 5-9. =

Martini X, Stelinski LL. 2017. Influence of abiotic factors on flight initiation by Asian citrus psyllid (Hemiptera: Liviidae). Environmental Entomology 46(2): 369-375.

Ma ZF, Liu J, Yang SQ. 2013. Climate change in southwest China during 1961-2010: impacts and adaptation. Advances in Climate Change Research 4(4):223-229.

Narouei-Khandan HA, Halbert SE, Worner SP, van Bruggen AHC. 2016. Global climate suitability of citrus huanglongbing and its vector, the Asian citrus psyllid, using two correlative species distribution modeling approaches, with emphasis on the USA. European Journal of Plant Pathology 144(3): 655-670.

Peer] reviewing PDF | (2019:01:34434:2:1:NEW 1 Jun 2019) 
564 Onagbola EO, Meyer WL, Boina DR, Stelinski LL. 2008. Morphological characterization of the

565

566

567

568

569

570

571

572

573

574

575

576

577

578

579

580

581

582

583

584

585

586

587

588

589

590

591

592

593

594

595

596

597

598

599

600

601

602

603

604

605

606

607

608

609 antennal sensilla of the Asian citrus psyllid, Diaphorina citri kuwayama (Hemiptera: Psyllidae), with reference to their probable functions. Micron 39(8): 1184-1191.

Petitpierre B, Kueffer C, Broennimann O, Randin C, Daehler C, Guisan A. 2012. Climatic niche shifts are rare among terrestrial plant invaders. Science 335(6074): 1344-1348.

Penado A, Rebelo H, Goulson D. 2016. Spatial distribution modelling reveals climatically suitable areas for bumblebees in undersampled parts of the Iberian Peninsula. Insect Conservation and Diversity 9(5): 391-401.

Phillips SJ, Dudík M, Schapire RE. 2019. [Internet] Maxent software for modeling species niches and distributions (Version 3.4.1). Available from url: http://biodiversityinformatics.amnh.org/open_source/maxent/. Accessed on 2019-2-26.

Pacifici M, Visconti P, Butchart SHM, Watson JEM, Cassola F, Rondinini C. 2017. Species' traits influenced their response to recent climate change. Nature Climate Change 7(3): 205208.

Qin JL, Yang XH, Yang ZW Luo JT, Lei XF. 2017. New technology for using meteorological information in forest insect pest forecast and warning systems. Pest management science 73(12): 2509-2518.

Rwomushana I, Khamis FM, Grout TG, Mohamed SA, Sétamou M, Borgemeister C, Heya HM, Tanga CM, Nderitu PW, Seguni ZS, Materu CL, Ekesi S. 2017. Detection of Diaphorina citri Kuwayama (Hemiptera: Liviidae) in Kenya and potential implication for the spread of huanglongbing disease in East Africa. Biological Invasions 19(10): 2777-2787.

Ruan CQ, Chen JL, Liu B, Duan YP, Xia YL. 2012. Morphology and behavior of Asian citrus psyllid, Diaphorina citri Kuwayama. Chinese Agricultural Science Bulletin 28(31): 186190.

Ren SL, Ou D, Zhang LH, Sang W, Ji QH, Qiu BL. 2018. Effects of different host plants on the development and reproduction of the Asian citrus psyllid Diaphorina citri. Chinese Journal of Applied Entomology 55(4): 602-607.

Song Y, Luo YF. 2017. Advances in the transmission characteristics and mechanism of Candidatus Liberobacter asiaticus by Diaphorina citri Kuwayama (Hemiptera:Liviidae). Journal of Environmental Entomology 39(4): 955-962.

Sule H, Muhamad R, Omar D, Hee AKW, Zazali C. 2012. Dispersion pattern and sampling of Diaphorina citri Kuwayama (Hemiptera: Psylidae) populations on citrus suhuiensis hort. Ex Tanaka in Padang Ipoh Terengganu, Malaysia. Pertanika Journal of Tropical Agricultural Science, 35: 25-35.

Shimwela MM, Narouei-Khandan HA, Halbert SE, Keremane ML, Minsavage GV, Timilsina S, van Bruggen AH. 2016. First occurrence of Diaphorina citri in East Africa, characterization of the Ca. Liberibacter species causing huanglongbing (HLB) in Tanzania, and potential further spread of D. citri and HLB in Africa and Europe. European Journal of Plant Pathology 146(2): 349-368.

Tsai JH, Liu YH. 2000. Biology of Diaphorina citri (Homoptera: Psyllidae) on four host plants. Journal of Economic Entomology 93(6): 1721-1725.

Wang YJ, Xu CB, Tian MY, Deng X, Cen Y, He Y. 2017. Genetic diversity of Diaphorina citri and its endosymbionts across east and south-east Asia. Pest Management Science, 73(10): 2090-2099.

Wang YJ, Lu JM, Beattie GAC, Islam MR, Om N, Dao HT, Van NL, Zaka SM, Guo J, Tian M, Deng X, Tan S, Holford P, He Y, Cen Y. 2018a. Phylogeography of Diaphorina citri 
610

611

612

613

614

615

616

617

618

619

620

621

622

623

624

625

626

627

628

629

630

631

632

633

634

635

636

637

638

639

640

641

642

643

644

645

646

647

648

649

650

651

652

653

654

655

(Hemiptera: Liviidae) and its primary endosymbiont, 'Candidatus Carsonella ruddii': an evolutionary approach to host-endosymbiont interaction. Pest Management Science 74(9): 2185-2194.

Wang XL, Li XN, Feng XD, Wu LF. 2016. Investigation on the psyllid, Diaphorina cirtri and huanglongbing in China. Plant Quarantine 30(2): 44.

Wang QZ, Liu YM, Li SM, Zhao Y, Wang W. 2018b. Chemical composition of essential oil of the invasive plant Praxelis clematidea and its repellence and lethality to Diaphorina citri. Chinese Journal of Applied Entomology 55(1):117-125.

Wang RL, Li Q, He SS, Liu Y, Wang MT, Jiang G. 2018c. Modeling and mapping the current and future distribution of Pseudomonas syringae pv. actinidiae under climate change in China. Plos One 13(2): e0192153.

Wang YS, Xie BY, Wan FH, Xiao QM, Dai LY. 2007. Application of ROC curve analysis in evaluating the performance of alien species potential distribution models. Biodiversity Science 15(4): 365-372.

Wang C, Hawthorne D, Qin YJ, Pan XB, Li ZH, Zhu SF. 2017b. Impact of climate and host availability on future distribution of Colorado potato beetle. Scientific Reports 7(1): 4489.

Wang SQ, Xiao YL, Zhang HY. 2015. Studies of the past, current and future potential distributions of Diaphorina citri Kuwayama (Homoptera: Psyllidae) in China. Chinese Journal of Applied Entomology 52(5): 1140-1148.

$\mathrm{Wu}$ Y. 2018. Technical efficiency evaluation and influencing factors analysis of citrus planting in China. Chinese Journal of Agricultural Resources and Regional Planning 39(9): 99-107.

Xiang Y, Qi CJ, Lu Q. 2014. The comparative advantage and its influencing factors of citrus production in Hubei province. Economic Geography 34(11): 134-139.

Yao TS, Zhou Y, Zhou CY. 2018. Advances in researches on the occurrence and control of Asia citrus psyllid. Journal of Fruit Science 35(11): 1413-1421.

Yu X, Killiny N. 2018. The secreted salivary proteome of Asian citrus psyllid Diaphorina citri. Physiological Entomology 43(4): 324-333.

Yang YP, Huang MD, C Beattie G A, Xia YL, Ouyang GC, Xiong JJ. 2006. Distribution, biology, ecology and control of the psyllid Diaphorina citri Kuwayama, a major pest of citrus: a status report for China. International Journal of Pest Management 52(4): 343-352.

Zhang L, Huo ZG, Wang L, Jiang YY. 2012. Effects of climate change on the occurrence of crop insect pests in China. Chinese Journal of Ecology 31(6):1499-1507.

Zhang HT, Luo D, Mu XD, Xu M, Wei H, Luo JR, Zhang JE, Hu YC. 2016. Predicting the potential suitable distribution area of the apple snail Pomacea canaliculata in China based on multiple ecological niche models. Chinese Journal of Applied Ecology 27(4): 12771284.

Zhang TJ, Liu G. 2017. Study of methods to improve the temporal transfer ability of niche model. Journal of China Agricultural University 22(2): 98-105.

Zhu YJ, W W, Li H, Wang BZ, Yang XH, Liu YS. 2018. Modelling the potential distribution and shifts of three varieties of Stipa tianschanica in the eastern Eurasian Steppe under multiple climate change scenarios. Global Ecology and Conservation 16: e00501.

Zhong JH. 2010. Heat tolerance of Coccobius azumai Tachikawa adult. Journal of Fujian Agriculture and Forestry University (Natural Science Edition) 39(5): 465-470.

Zhang HN, Lv JH, Ya CC, Ma D, Bai XG. 2014. Efftect of high-temperature treatment on mortality and moisture content of Sitophilus Zeamais. Journal of Henan University of Technology (Natural Science Edition) 35(6): 45-48.

PeerJ reviewing PDF | (2019:01:34434:2:1:NEW 1 Jun 2019) 
656 Zhao HY, Guo JQ, Zhang CJ, Sun LD, Zhang XD, Lin JJ, WangYH, Fang F, Ma PL, Liu CH, Li 657 YC, Fang JG, Li L, Li HM, Zheng GF, Deng ZY, Dong AX. 2014. Climate change impacts 658 and adaptation strategies in northwest China. Advances in Climate Change Research 5(1):765916. 
Figure 1

ROC curve and AUC values for the initial model (a) and the final model (b)
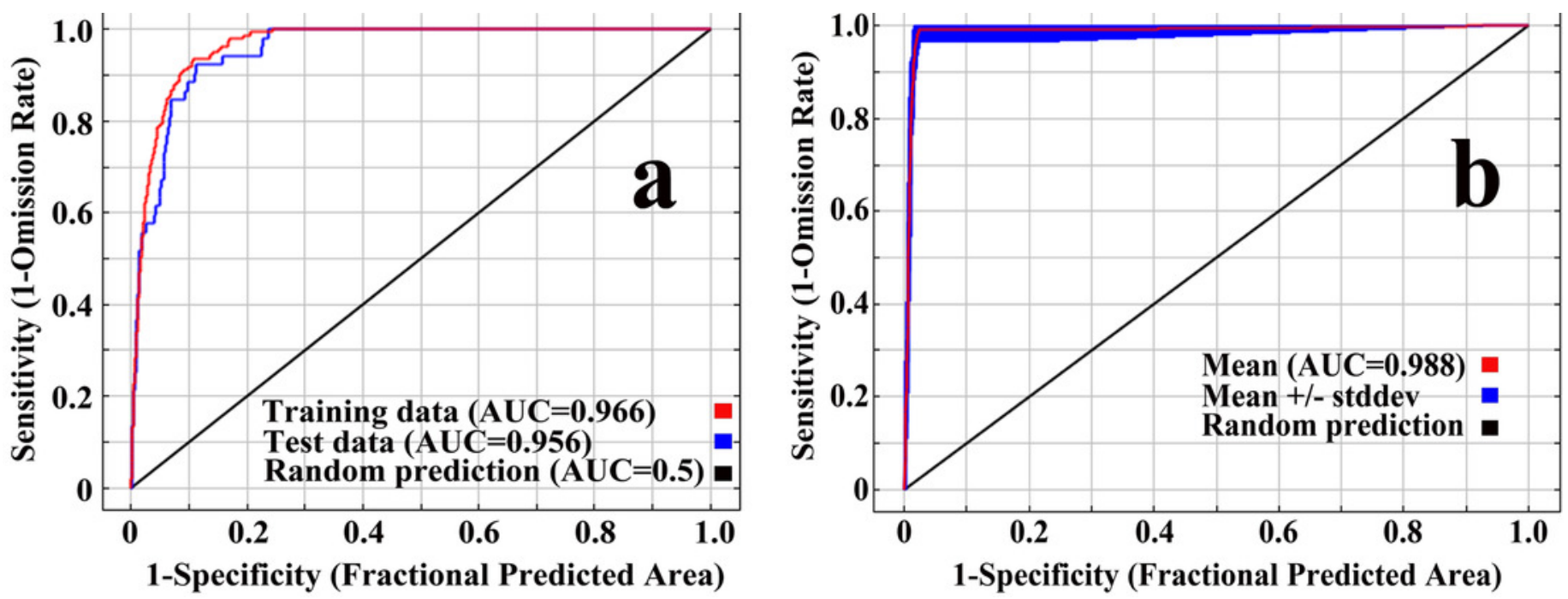
Figure 2

Probability $(P)$ of the potential distribution of $D$. citri in China based on the MaxEnt model.

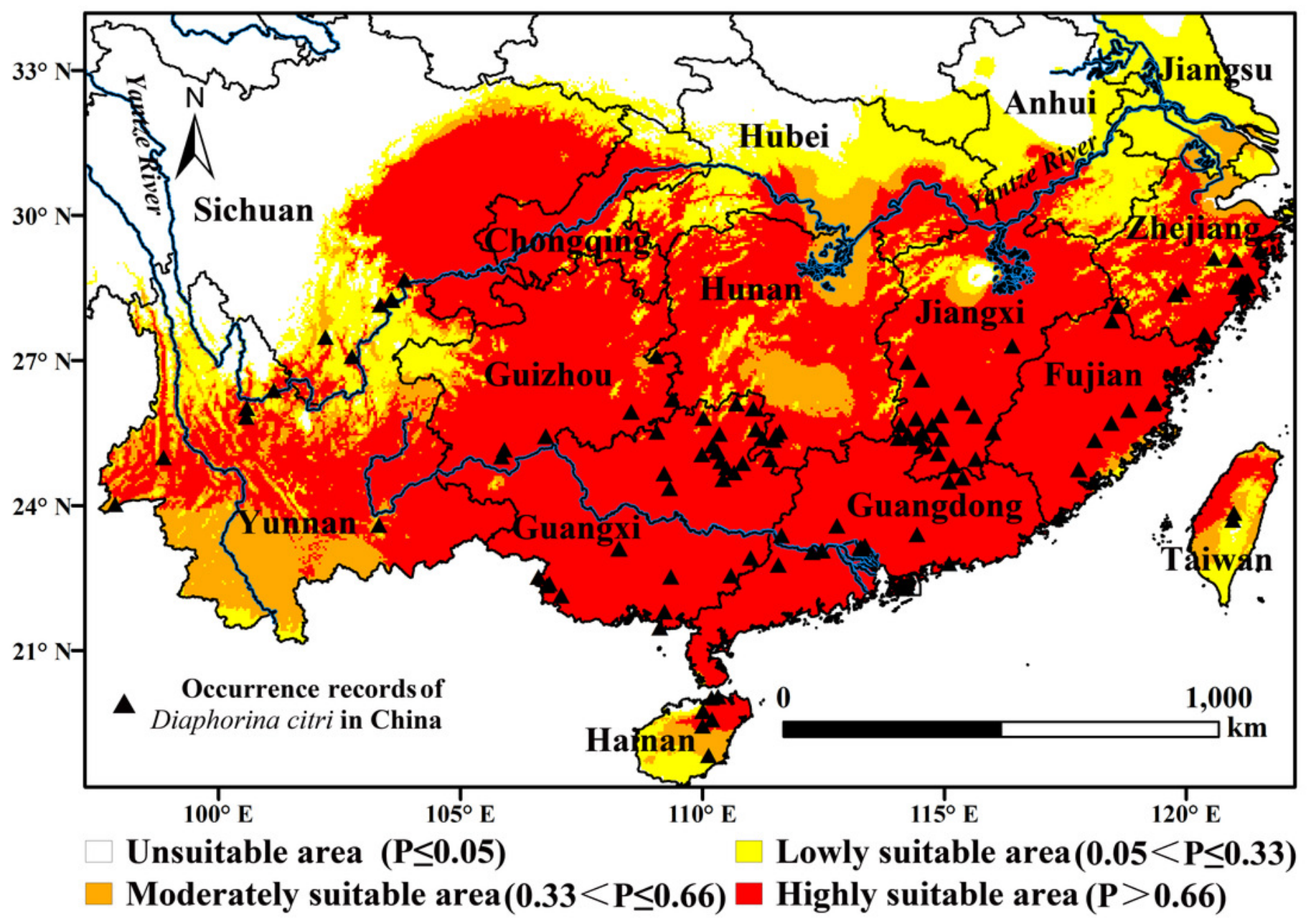




\section{Figure 3}

Jackknife test for variable importance in the $D$. citri suitability distribution: values shown are averages over 10 replicate runs.

With only variables Without variables $\square$ With all variables

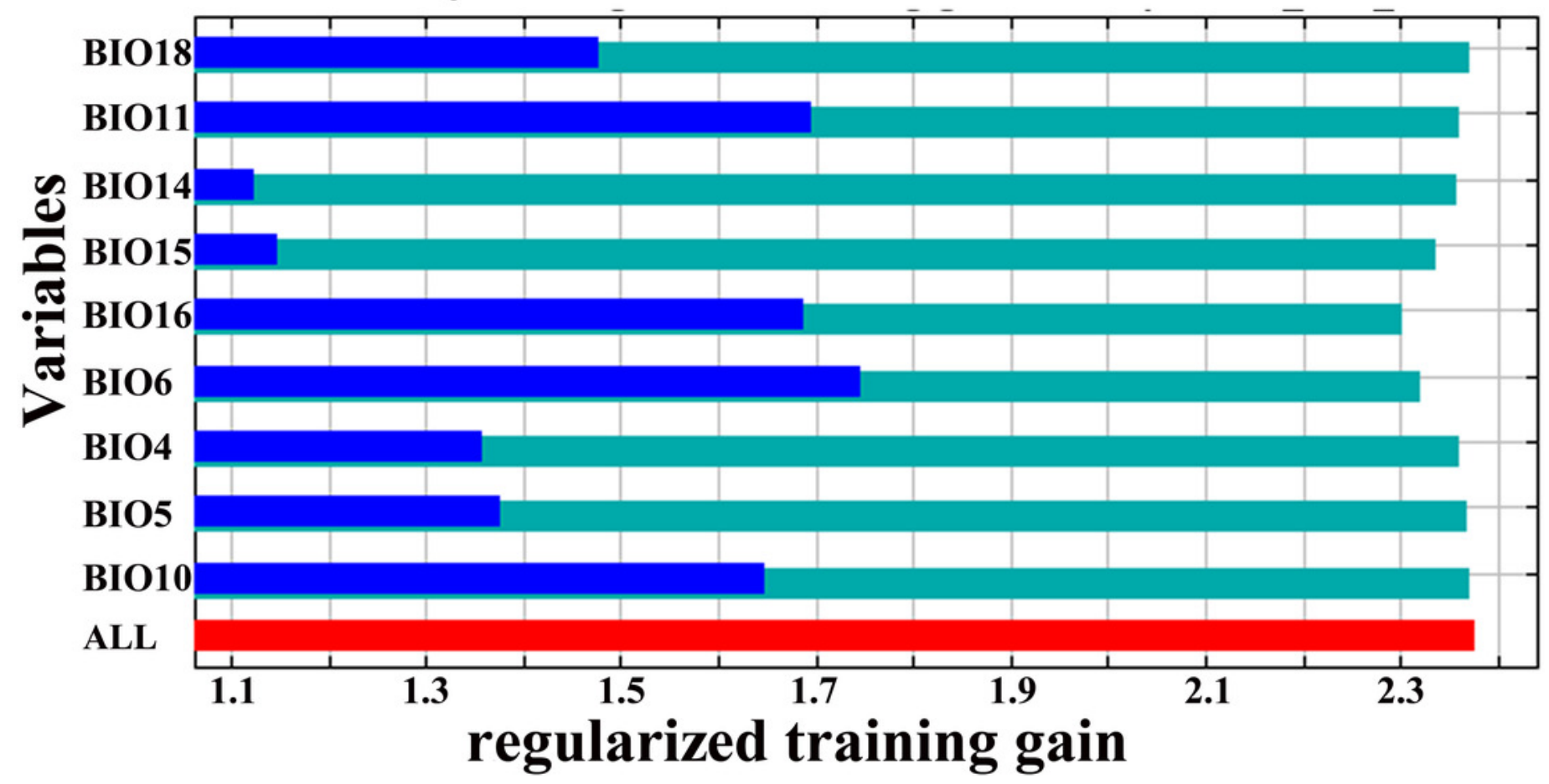


Figure 4

Response curves of environmental variables in the MaxEnt models: (A) BIO6; (B) BIO11; . (C) BIO16; (D) BIO18; (E) BIO5; (F) BIO4; (G) BIO10. 

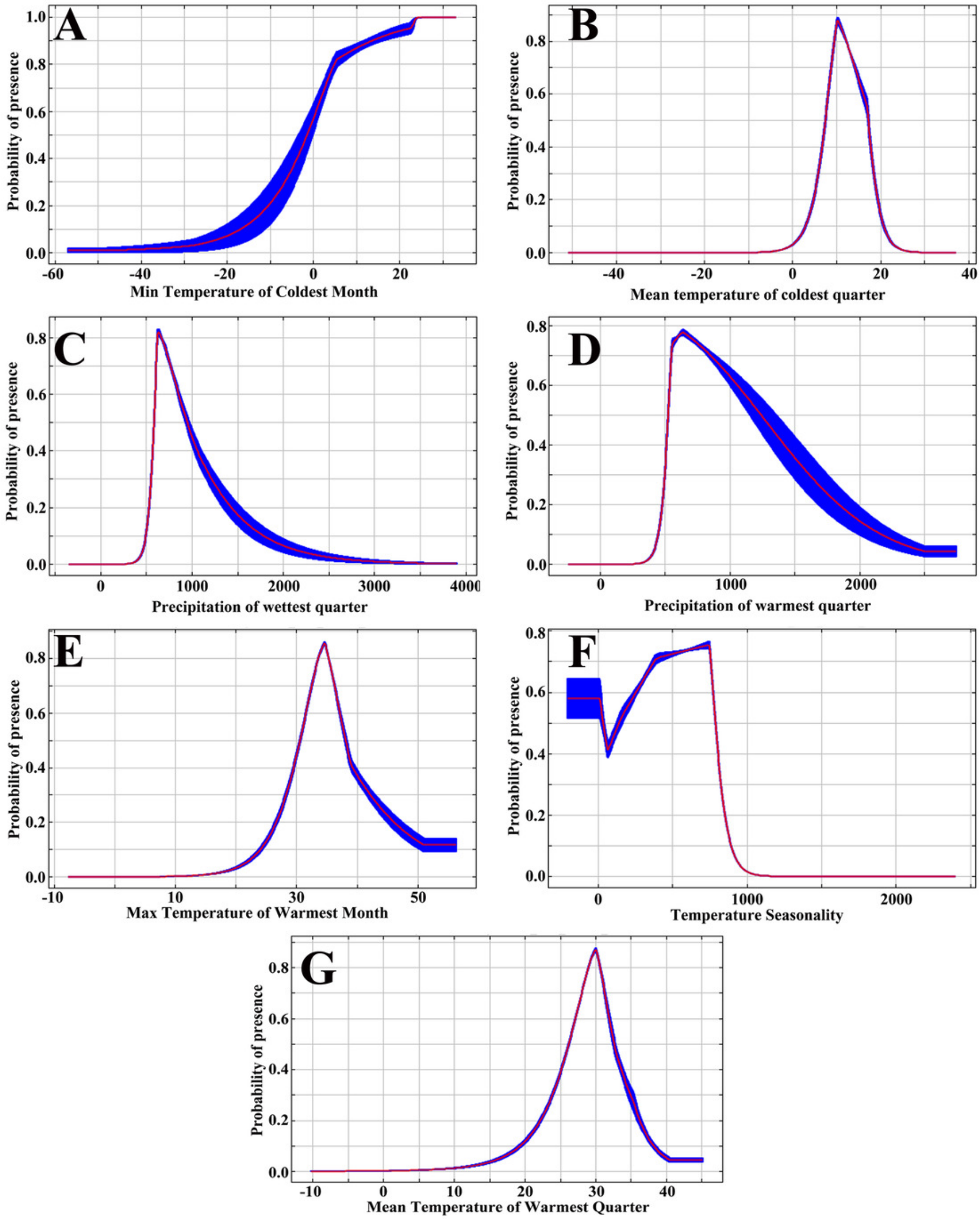


\section{Table $\mathbf{1}$ (on next page)}

Percent contribution and cumulative contribution of the environmental variables to the Maxent model. 
1 Table 1:

2 Percent contribution and cumulative contribution of the environmental variables to the Maxent model.

\begin{tabular}{|l|l|l|l|}
\hline Environmental variables & Code & $\begin{array}{l}\text { Percent } \\
\text { contribution }\end{array}$ & $\begin{array}{l}\text { Cumulative } \\
\text { importance }\end{array}$ \\
\hline Mean Temperature of Coldest Quarter & BIO11 & 33.7 & 33.7 \\
\hline Precipitation of warmest quarter & BIO18 & 32.6 & 66.3 \\
\hline Temperature seasonality & BIO4 & 23.2 & 89.5 \\
\hline Precipitation of driest month & BIO14 & 2.6 & 92.1 \\
\hline Precipitation seasonality & BIO15 & 1.6 & 93.7 \\
\hline Min Temperature of Coldest Month & BIO6 & 1.3 & 95 \\
\hline Precipitation of Wettest Quarter & BIO16 & 1.2 & 96.2 \\
\hline Mean Temperature of Warmest Quarter & BIO10 & 1.0 & 97.2 \\
\hline Precipitation of Driest Quarter & BIO17 & 0.6 & 97.8 \\
\hline Annual Mean Temperature & BIO1 & 0.5 & 98.3 \\
\hline Precipitation of Wettest Month & BIO13 & 0.3 & 98.6 \\
\hline Isothermality (BIO2/BIO7) (* 100) & BIO3 & 0.3 & 98.9 \\
\hline Mean Diurnal Range (Mean of monthly & BIO2 & 0.2 & 99.1 \\
\hline Precipitation of Coldest Quarter & BIO19 & 0.2 & 99.3 \\
\hline Mean Temperature of Wettest Quarter & BIO8 & 0.2 & 99.5 \\
\hline Max Temperature of Warmest Month & BIO5 & 0.2 & 99.7 \\
\hline Mean Temperature of Driest Quarter & BIO9 & 0.1 & 99.8 \\
\hline Altitude & Alt & 0.1 & 99.9 \\
\hline Temperature Annual Range (BIO5-BIO6) & BIO7 & 0.1 & 100 \\
\hline Annual Precipitation & BIO12 & 0 & 100 \\
\hline & & & \\
\hline
\end{tabular}

3

4

5 